\title{
CD47-blocking immunotherapies stimulate macrophage-mediated destruction of small-cell lung cancer
}

\author{
Kipp Weiskopf, ${ }^{1,2,3}$ Nadine S. Jahchan, ${ }^{3,4,5}$ Peter J. Schnorr, ${ }^{1,2,3}$ Sandra Cristea, ${ }^{3,4,5}$ Aaron M. Ring, ${ }^{1,2,3,6,7}$ Roy L. Maute, ${ }^{1,2,3}$ \\ Anne K. Volkmer, ${ }^{1,2,3,8}$ Jens-Peter Volkmer, ${ }^{1,2,3}$ Jie Liu, ${ }^{1,2,3}$ Jing Shan Lim, ${ }^{3,4,5}$ Dian Yang, ${ }^{3,4,5}$ Garrett Seitz, ${ }^{3,4,5}$ Thuyen Nguyen, ${ }^{3,4,5}$ \\ Di Wu, ${ }^{2,3,6,7}$ Kevin Jude, ${ }^{2,3,6,7}$ Heather Guerston, ${ }^{9}$ Amira Barkal, ${ }^{1,2,3}$ Francesca Trapani, ${ }^{10}$ Julie George, ${ }^{11}$ John T. Poirier, ${ }^{12}$ \\ Eric E. Gardner, ${ }^{12}$ Linde A. Miles, ${ }^{12}$ Elisa de Stanchina, ${ }^{12}$ Shane M. Lofgren, ${ }^{3,4,5}$ Hannes Vogel, ${ }^{3,13}$ Monte M. Winslow, ${ }^{5,13}$ \\ Caroline Dive, ${ }^{10}$ Roman K. Thomas, ${ }^{11,14}$ Charles M. Rudin, ${ }^{12}$ Matt van de Rijn, ${ }^{13}$ Ravindra Majeti, ${ }^{1,2,3}$ K. Christopher Garcia, ${ }^{2,3,6,7}$ \\ Irving L. Weissman, ${ }^{1,2,3,13}$ and Julien Sage ${ }^{3,4,5}$
}

IInstitute for Stem Cell Biology and Regenerative Medicine, ${ }^{2}$ Ludwig Center for Cancer Stem Cell Research and Medicine, ${ }^{3}$ Stanford Cancer Institute, ${ }^{4}$ Department of Pediatrics, ${ }^{5}$ Department of Cenetics, ${ }^{6}$ Department of Molecular and Cellular Physiology, and Department of Structural Biology, and ${ }^{7}$ Howard Hughes Medical Institute, Stanford University School of Medicine, Stanford, California, USA. ${ }^{8}$ Department of Obstetrics and Gynecology, University of Düsseldorf, Düsseldorf, Germany. ${ }^{~}$ Blood and Marrow Transplantation, Stanford University School of Medicine, Stanford, California, USA. ${ }^{10}$ Clinical and Experimental Pharmacology Group, Cancer Research UK Manchester Institute, University of Manchester and Manchester Cancer Research Centre, Manchester, United Kingdom. "Department of Translational Cenomics, Center of Integrated Oncology Cologne-Bonn, Medical Faculty, University of Cologne, Cologne, Germany, and German Cancer Research Center (DKFZ), German Cancer Consortium (DKTK), Heidelberg, Germany. ${ }^{12}$ Memorial Sloan Kettering Cancer Center, New York, New York, USA. ${ }^{13}$ Department of Pathology, Stanford University Medical Center, Stanford, California, USA. ${ }^{14}$ Department of Pathology, University Hospital Cologne, Cologne, Germany.

\begin{abstract}
Small-cell lung cancer (SCLC) is a highly aggressive subtype of lung cancer with limited treatment options. CD47 is a cellsurface molecule that promotes immune evasion by engaging signal-regulatory protein alpha (SIRPa), which serves as an inhibitory receptor on macrophages. Here, we found that CD47 is highly expressed on the surface of human SCLC cells; therefore, we investigated CD47-blocking immunotherapies as a potential approach for SCLC treatment. Disruption of the interaction of CD47 with SIRP $\alpha$ using anti-CD47 antibodies induced macrophage-mediated phagocytosis of human SCLC patient cells in culture. In a murine model, administration of CD47-blocking antibodies or targeted inactivation of the $\mathrm{Cd} 47$ gene markedly inhibited SCLC tumor growth. Furthermore, using comprehensive antibody arrays, we identified several possible therapeutic targets on the surface of SCLC cells. Antibodies to these targets, including CD56/neural cell adhesion molecule (NCAM), promoted phagocytosis in human SCLC cell lines that was enhanced when combined with CD47-blocking therapies. In light of recent clinical trials for CD47-blocking therapies in cancer treatment, these findings identify disruption of the CD47/SIRP $\alpha$ axis as a potential immunotherapeutic strategy for SCLC. This approach could enable personalized immunotherapeutic regimens in patients with SCLC and other cancers.
\end{abstract}

\section{Introduction}

Small-cell lung cancer (SCLC), which originates from neuroendocrine cells of the lung $(1,2)$, is one of the most lethal subtypes of cancer. Each year, more than 25,000 patients are diagnosed with SCLC in the United States alone, and these patients typically live only 6-12 months after diagnosis. The 5-year survival

Authorship note: I.L. Weissman and J. Sage contributed equally to this work. Conflict of interest: The authors declare US patent applications pertaining to CD47blocking therapies for human disease, including "Targeted therapy for small cell lung cancer" (PCT/US2015/010650; K. Weiskopf, I.L. Weissman, J. Sage) and "Humanized and chimeric monoclonal antibodies to CD47" (PCT/US2011/036535; J. Liu, R. Majeti, I.L. Weissman). K. Weiskopf, A.M. Ring, J.P. Volkmer, J. Liu, R. Majeti, and I.L. Weissman declare consulting and/or equity ownership in Forty Seven Inc. R. Majeti and I.L. Weissman are directors of Forty Seven Inc. K. Weiskopf, A.M. Ring, and K.C. Garcia declare consulting and/or equity ownership in Alexo Therapeutics Inc.

Submitted: February 20, 2015; Accepted: April 29, 2016

Reference information: / Clin Invest. 2016;126(7):2610-2620. doi:10.1172/JCI81603. rate has remained dismal, hovering around 5\% since the 1970 s. Except for the combination of radiation and chemotherapy, there have been no new therapeutic approaches implemented in the clinic in the past few decades. Despite a plethora of clinical trials and substantial effort from many groups to identify novel treatment options, no targeted therapies have been approved for SCLC. SCLC is strongly linked to heavy cigarette smoking, and the continued increase in smokers worldwide suggests the prevalence of SCLC will increase in the future $(3,4)$. For these reasons, there is a dire need to identify novel therapeutic targets and generate new treatments for patients with SCLC.

One of the most promising advances in the field of oncology is immunotherapy, which aims to stimulate a patient's own immune system to attack and eliminate cancer cells. As tumors develop, they acquire mechanisms to avoid destruction by the immune system; understanding these mechanisms can lead to the development of new strategies that provoke the immune system to rec- 
A
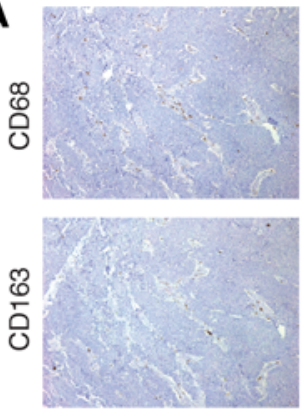

Low
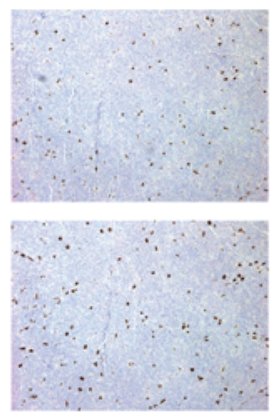

Moderate
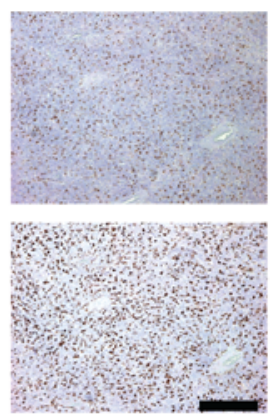

Intense

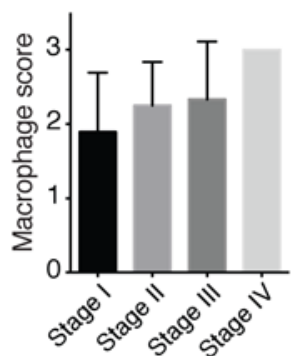

B

B SCLC cell lines

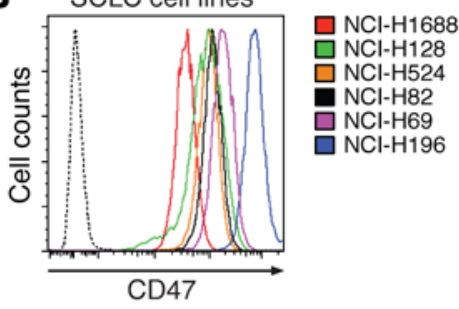

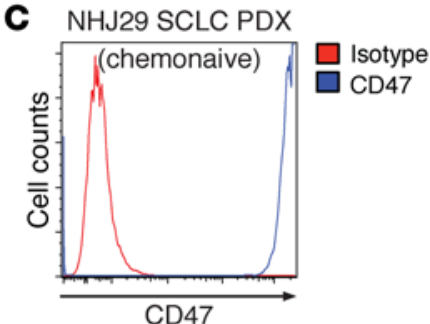

E Macrophage $\mathrm{NCl}-\mathrm{H} 82$

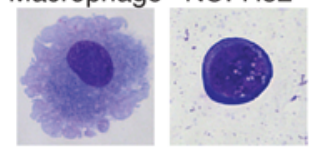

$\mathrm{NCl}-\mathrm{H} 82+$ Macrophages

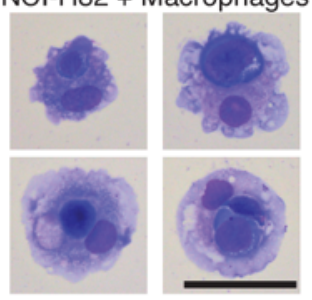

SCLC PDX

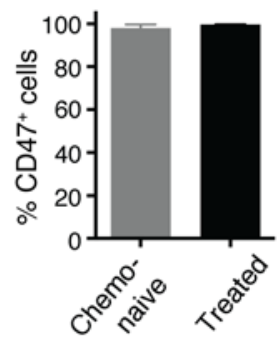

D

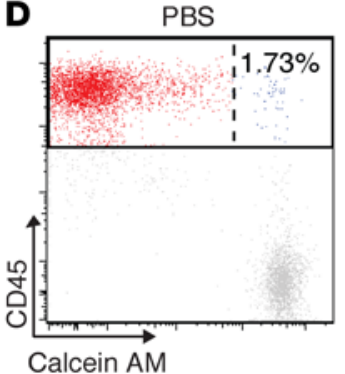

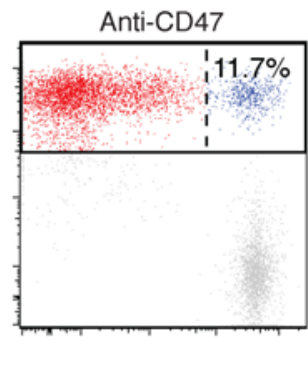
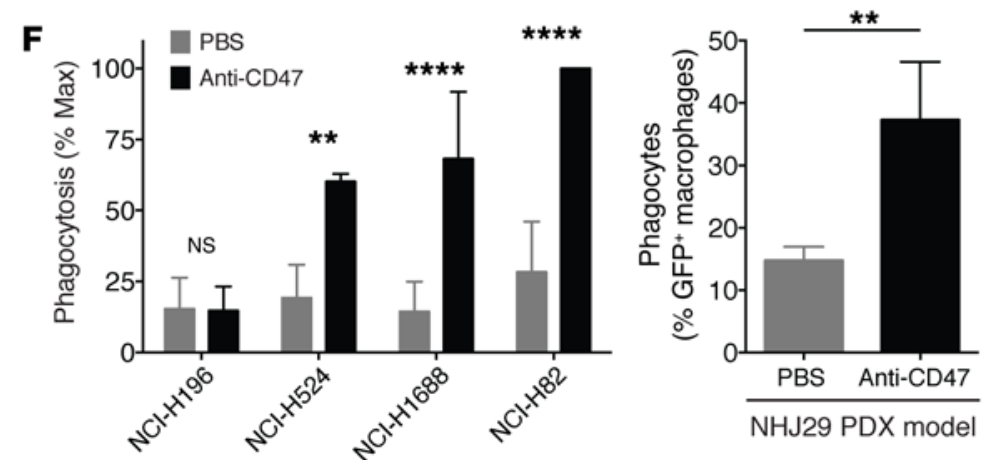

Figure 1. CD47 is a therapeutic target for SCLC. (A) Histological analysis of macrophage infiltration in SCLC patient samples $(n=79)$. Specimens were stained for the macrophage markers CD68 and CD163. Samples were scored from 1 to 3 (1, low; 2, moderate; 3, intense) based on macrophage infiltration, with representative images of each score depicted (left). One sample exhibited no evidence of macrophage infiltration (not shown). Chart depicts a summary of macrophage infiltration scores as varied by tumor stage (right) (correlation coefficient $r=0.2721 ; P=0.0153$ ). Scale bar: $300 \mu$ m. (B) CD47 expression on human SCLC cell lines $(n=6)$ by flow cytometry. Dotted black line, unstained NCI-H82 cells. (C) CD47 expression on SCLC patient sample PDX NJH29 (left) and quantification of CD47 on PDX samples from chemonaive patients $(n=3)$ and patients with recurrent tumors after chemotherapy (treated, $n=4$ ). (D) Gating strategy used for flow cytometry analysis of phagocytosis assays performed with human macrophages (CD45 ${ }^{+}$) and calcein AM-labeled SCLC cells. Percentages of calcein $\mathrm{AM}^{+}$macrophages out of total CD45+ macrophage population are indicated. (E) Representative images of cell populations after sorting. The double-positive population contains macrophages with engulfed tumor cells. Scale bar: $20 \mu \mathrm{m}$. Experiment performed twice with similar results. (F) Summary of phagocytosis assays using human macrophages and calcein AM-labeled SCLC cell lines (left) or primary NJH29 SCLC cells as analyzed by flow cytometry. SCLC cells were treated with vehicle control (PBS) or anti-CD47 antibodies (Hu5F9-C4). Assays performed with macrophages from independent donors $(n=4)$ and depicted as the percentages of calcein $\mathrm{AM}^{+}$macrophages (right) or normalized to the maximal response by each donor (left). Data represent mean $\pm \mathrm{SD}$. ${ }^{* *} P<0.01$; ${ }^{* * *} P<0.0001,2$-way ANOVA with Šidák correction (left) or 2-tailed $t$ test (right).

ognize cancer as foreign $(5,6)$. Previous studies have identified CD47, a cell-surface molecule, as a "marker of self" that prevents cells of the innate immune system from attacking hematologic malignancies and certain types of solid tumors (7-9). CD47 acts by sending inhibitory signals through SIRP $\alpha$, a receptor expressed on the surface of macrophages and other myeloid cells. In this sense, the CD47/SIRPa interaction serves as a myeloid-specific immune checkpoint, and blocking the interaction lowers the threshold for macrophage phagocytosis of cancer $(10,11)$. In most cases, an additional prophagocytic stimulus is necessary to induce phago- cytosis, which can be delivered by agents that contain Fc chains that engage activating $\mathrm{Fc}$ receptors on macrophages $(10,12)$. AntiCD47 antibodies or SIRP $\alpha$-Fc fusion proteins block CD47 and provide an Fc receptor stimulus; therefore, they can stimulate phagocytosis as single agents $(8,10,12,13)$. On the other hand, pure CD47 antagonists that lack Fc chains, such as high-affinity SIRP $\alpha$ monomers, augment phagocytosis only when combined with a tumor-binding antibody (10). By allowing maximal signaling from Fc receptors, CD47-blocking therapies have been shown to synergize with a variety of therapeutic antibodies for cancer $(10,12,14)$. 
In this study, we hypothesized that anti-CD47 agents might be efficacious as immunotherapies for SCLC. We found that SCLC cells express high levels of CD47 and that blocking CD47 enhances phagocytosis of SCLC cells and inhibits tumor growth. Since no therapeutic antibodies have been approved for SCLC, we aimed to identify antigens on the surface of SCLC cells and target them with monoclonal antibodies in combination with CD47-blocking therapies to achieve maximal antitumor responses against SCLC. Through this approach, we identified immunotherapeutic strategies that could be applied to the treatment of SCLC.

\section{Results}

Macrophages are present in SCLC tumors, and CD47 is expressed on the surface of SCLC cells. Some evidence suggests that, in conjunction with a high number of T cells and small tumor size, high macrophage counts correlate with favorable survival in SCLC patients (15). We examined macrophage infiltration into SCLC tumors by staining 79 SCLC patient samples for the macrophage markers CD68 and CD163 (Figure 1A). Overall, 98.7\% (78/79) of samples exhibited evidence of macrophage infiltration, with the majority of samples $(77.2 \%, 61 / 79)$ showing moderate or intense infiltration. Our analysis indicated a positive association between macrophage infiltration and tumor stage (Figure 1A). To examine molecular signatures of macrophage infiltration, we analyzed the expression of macrophage-specific markers in recent RNA sequencing (RNA-seq) data sets from bulk human tumors $(16,17)$. We found that human SCLC biopsies expressed significant RNA levels for the macrophage markers CD68, CD163, CD14, and the CD47 receptor SIRPa (Supplemental Figure 1A; supplemental material available online with this article; doi:10.1172/JCI81603DS1). On the other hand, microarray expression analysis suggested that established cell lines did not substantially express these macrophage markers relative to control genes (Supplemental Figure 1B). Together, these data confirm that human SCLC tumors contain a significant fraction of macrophages in vivo. These cells could act as effectors against cancer in response to agents targeting CD47.

To further evaluate whether CD47-blocking therapies could be applied to SCLC, we examined CD47 expression in human SCLC cells. We found that CD47 mRNA expression was high in both bulk tumors and cell lines (Supplemental Figure 1, A and B). A prior study also demonstrated higher CD47 mRNA expression in SCLC samples and neuronal/neuroendocrine samples relative to other normal tissues (18). Flow cytometry analysis showed that 6 of 6 human SCLC cell lines tested expressed high levels of the CD47 protein on the cell surface (Figure 1B). Importantly, we found high expression of CD47 both in chemonaive and chemoresistant patient-derived xenografts (PDXs) obtained from SCLC patients and exclusively passaged in immunocompromised mice (Figure 1C and Supplemental Figure 2A). Similarly, most SCLC cells in xenografts derived from circulating tumor cells (CTCs) isolated from the blood of 4 SCLC patients expressed CD47 (19) (Supplemental Figure 2B).

CD47-blocking antibodies induce phagocytosis of SCLC cells by human macrophages. To validate CD47 as a genuine therapeutic target in SCLC, we next performed in vitro phagocytosis assays. Primary human macrophages were cocultured with SCLC cells in the presence of a vehicle control or anti-CD47 antibodies. We tested anti-CD47 antibody Hu5F9-G4, a humanized antibody that blocks the interaction between CD47 and SIRP $\alpha$ (Supplemental Figure 3) (20) and is undergoing evaluation in phase I clinical trials (ClinicalTrials.gov NCT02216409, NCT02678338). Flow cytometry was used to measure phagocytosis (10), which was evaluated by the percentage of macrophages engulfing calcein acetoxymethyl-labeled (calcein AM-labeled) SCLC cells (Figure 1D). FACS was used to confirm that the double-positive population contained macrophages with engulfed tumor cells (Figure 1E). Four SCLC samples were subjected to evaluation in phagocytosis assays. Three cell lines (NCI-H524, NCI-1688, and NCI-H82) exhibited significant increases in phagocytosis when treated with the CD47-blocking antibody (Figure 1F). One cell line, NCI-H196 (a variant form of SCLC), appeared to be resistant to phagocytosis despite expressing high levels of CD47, suggesting that additional mechanisms modify the susceptibility of this cell line to macrophage attack (Figure 1F). The primary PDX NJH29 was also subjected to phagocytosis assays with human macrophages. Treatment of this sample with anti-CD47 antibodies resulted in a significant increase in phagocytosis (Figure 1F). We next examined the inhibitory effects of CD47 by genetic analysis. Using CRISPR/ Cas9 genome editing, we generated a CD47 knockout variant of NCI-H82 (Supplemental Figure 4A). This mutant cell line became unresponsive to treatment with Hu5F9-G4 when evaluated in phagocytosis assays, indicating that Hu5F9-G4 does not stimulate phagocytosis via off-target effects (Supplemental Figure 4B). Overall, these data indicate that blocking CD47 on human SCLC cells in culture can promote the phagocytosis of these tumor cells by human macrophages.

Structural analysis reveals the antagonistic mechanism of the anti-CD47 antibody Hu5F9-G4. To explore the mechanism of CD47 antagonism by anti-CD47 antibodies, we performed structural analysis of Hu5F9-G4 in complex with the CD47 extracellular domain (CD47-ECD). We were unsuccessful at cocrystallizing the CD47-ECD with Hu5F9-G4 in either Fab or scFv formats; therefore, we prepared a diabody from Hu5F9-G4 by fusing the heavy and light variable domains $\left(\mathrm{V}_{\mathrm{H}}\right.$ and $\left.\mathrm{V}_{\mathrm{L}}\right)$ with a short GGSGG linker. We crystallized the Hu5F9-G4 diabody/ CD47-ECD complex and solved the structure at $2.8 \AA$ resolution (Supplemental Table 1). The crystal structure revealed the Hu5F9-G4 diabodies form a symmetric, domain-swapped dimer linking 2 copies of the CD47-ECD. The interfaces were dominated by polar contacts; only $365 \AA^{2}$ and $310 \AA^{2}$ of CD47-ECD surface area were buried by the $\mathrm{V}_{\mathrm{H}}$ and $\mathrm{V}_{\mathrm{L}}$ domains, respectively. The $\mathrm{V}_{\mathrm{H}}$ of Hu5F9-G4 formed 6 hydrogen bonds to the CD47ECD via the CDR H1, H2, and H3 loops. The $\mathrm{V}_{\mathrm{L}}$ of Hu5F9-G4 interacted with the CD47-ECD and formed 5 hydrogen bonds via the CDR L1 and L2 loops (Figure 2, A-C).

Notably, the Hu5F9-G4 diabody/CD47-ECD complex bears a striking resemblance to that of SIRP $\alpha$ in complex with CD47 (21). Although the binding mode of SIRP $\alpha$ to CD47 was different, interacting via the SIRP $\alpha \mathrm{BC}, \mathrm{C}^{\prime} \mathrm{D}, \mathrm{DE}$, and FG loops and burying $950 \AA^{2}$ of surface area, the 2 proteins bound to overlapping epitopes of CD47 (Figure 2, B and C), including the N-terminal pyroglutamate (pGlu), the BC and FG loops, and the face of the central $\beta$-sheet. This finding indicates that Hu5F9-G4 and SIRP $\alpha$ compete for the same binding site on CD47, explaining the antagonistic properties of Hu5F9-G4. 
A

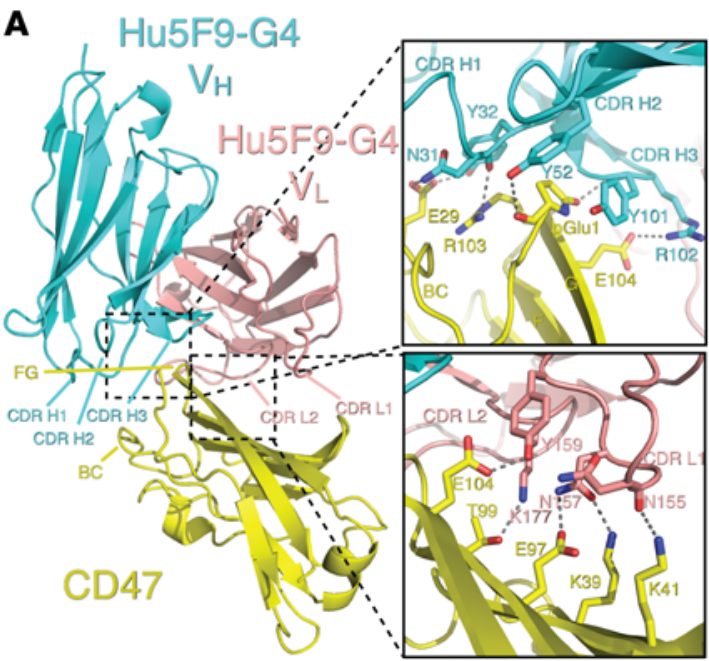

B

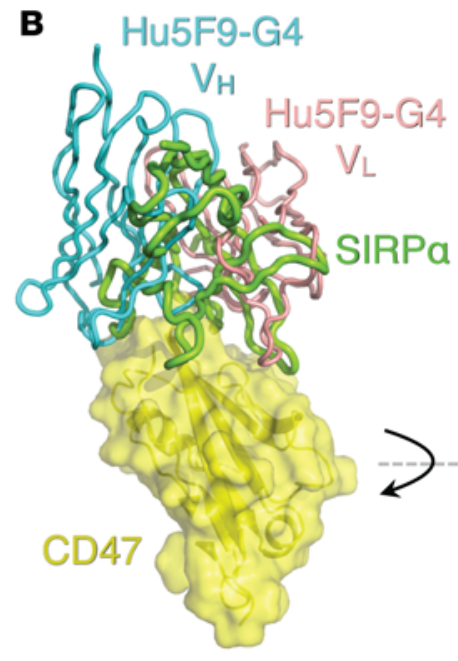

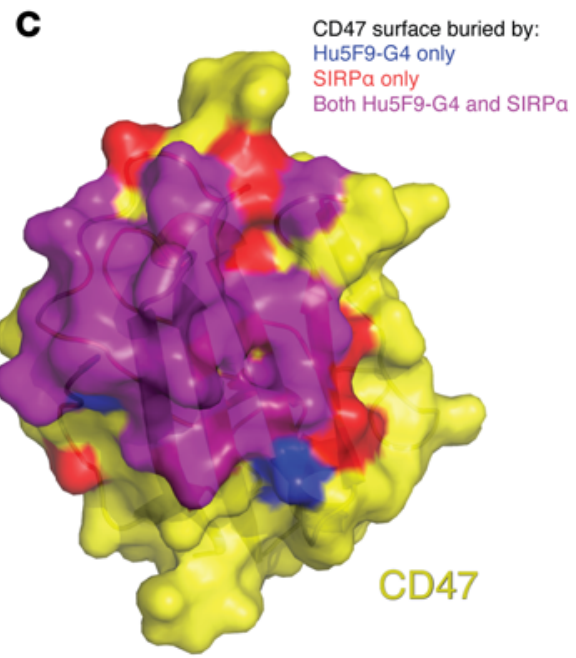

Figure 2. The crystal structure of Hu5F9-G4 diabody in complex with CD47 demonstrates competitive antagonism. (A) Crystal structure of CD47-ECD in complex with Hu5F9-G4 diabody (PDB 5IWL). For clarity, only 1 domain from each diabody chain is shown. The CDR1, CDR2, and CDR3 loops from the $V_{H}$ domain (cyan) and the CDR1 and CDR2 loops from the $V_{L}$ domain (pink) contribute to binding the CD47-ECD epitope. Contact residues are indicated in the inset. (B) Hu5F9-G4/CD47-ECD structure superimposed on SIRP $\alpha / C D 47-E C D$ (PDB ID 2JJS), demonstrating a shared binding interface. (C) CD47-binding interface showing residues interacting with only SIRP $\alpha$ (red), only Hu5F9-G4 (blue), or both ligands (purple).

CD47-blocking antibodies inhibit the growth of SCLC tumors in vivo. To evaluate the potential efficacy of CD47-blocking agents when administered as therapies for human SCLC in vivo, we engrafted fast-growing, metastatic NCI-H82 SCLC cells into the lower flanks of NSG mice. These immunocompromised mice lack functional T cells, B cells, and NK cells, but retain functional macrophages $(14,22)$. Approximately 1 week after engraftment, mice were randomized into treatment, with vehicle control or antiCD47 antibody Hu5F9-G4 administered every other day. After 2 weeks of treatment, a significant difference in median tumor volume was observed that persisted through the remainder of the experiment (Figure 3A). After approximately 1 month of treatment, the median tumor volume for the vehicle control cohort was $837.8 \mathrm{~mm}^{3}$ versus $160.2 \mathrm{~mm}^{3}$ for the cohort treated with the antiCD47 antibody $(P=0.0281)$ (Figure $3 \mathrm{~A})$. Therefore, the CD47blocking antibody significantly inhibited growth of SCLC tumors in this subcutaneous xenograft model.

We also tested the in vivo efficacy of CD47-blocking antibodies against a PDX, which more closely models treatment of patients $(23,24)$. Cells from the NJH29 PDX model were transduced to express GFP-luciferase to allow dynamic measurements of tumor growth in vivo. Tumors were engrafted subcutaneously into the lower flanks of mice and allowed to establish for approximately 2 weeks. Mice were then randomized into 2 cohorts treated with vehicle control or Hu5F9-G4 administered every other day. We found that treatment with the anti-CD47 antibody significantly inhibited tumor growth compared with control treatment, as assessed by tumor volume measurements and bioluminescence imaging (Figure 3, B-D). Treatment with the CD47-blocking therapy also produced striking benefits in survival (Figure 3E). By day 125 after engraftment, all mice in the control group had died, whereas the majority of mice in the anti-CD47 antibody group had only small tumors that failed to progress even after 225 days after engraftment. Histopathological analysis of control tumors and tumors treated with the anti-CD47 antibody showed prominent features of tumor cell death in the anti-CD47-treated group as well as increased presence of macrophages infiltrating the tumors (Supplemental Figure 5, A-F). The tumors that eventually grew in the anti-CD47 treatment group still expressed CD47 at their surface, indicating that the mechanism of resistance is not directly linked to decreased CD47 expression (Supplemental Figure 5G).

We next generated a GFP-luciferase ${ }^{+}$NCI-H82 cell line to establish an orthotopic model of SCLC in patients. We engrafted these GFP-luciferase ${ }^{+}$SCLC cells directly into the left thoracic cavities of mice. Four days after injections, engraftment was confirmed by bioluminescence imaging. We then randomized the mice into 2 cohorts treated with either vehicle control or Hu5F9G4 administered every other day. Postmortem analysis in both groups revealed that tumors had grown within the thoracic cavity or in the parathoracic region (Figure 4, A and B). Some mice exhibited spontaneous metastases to the liver (Figure 4A), which was not observed in the subcutaneous model; the liver is the most common site of distant metastases in SCLC patients (25). Monitoring tumor growth over time by bioluminescence imaging, we found that in this context, the CD47-blocking antibody also produced a significant inhibition of tumor growth (Figure 4, C and D). Additionally, we observed a significant benefit in survival for the cohort treated with the CD47-blocking antibody (Figure 4E). Thus, the anti-CD47 strategy is also effective against human SCLC tumors growing in the thoracic cavity of mice, further indicating that CD47-blocking agents inhibit SCLC growth and could be effective therapies for patients with SCLC.

To evaluate the effects of CD47 deletion by genetic analysis, we evaluated the growth of the NCI-H82 CD47 knockout cell line in NSG mice. Although these cells exhibited no defects in proliferation in vitro (Supplemental Figure 4C), we observed a significant inhibition of tumor growth over time relative to control cells when engrafted in vivo (Figure 5A). The NCI-H82 CD47 knockout 

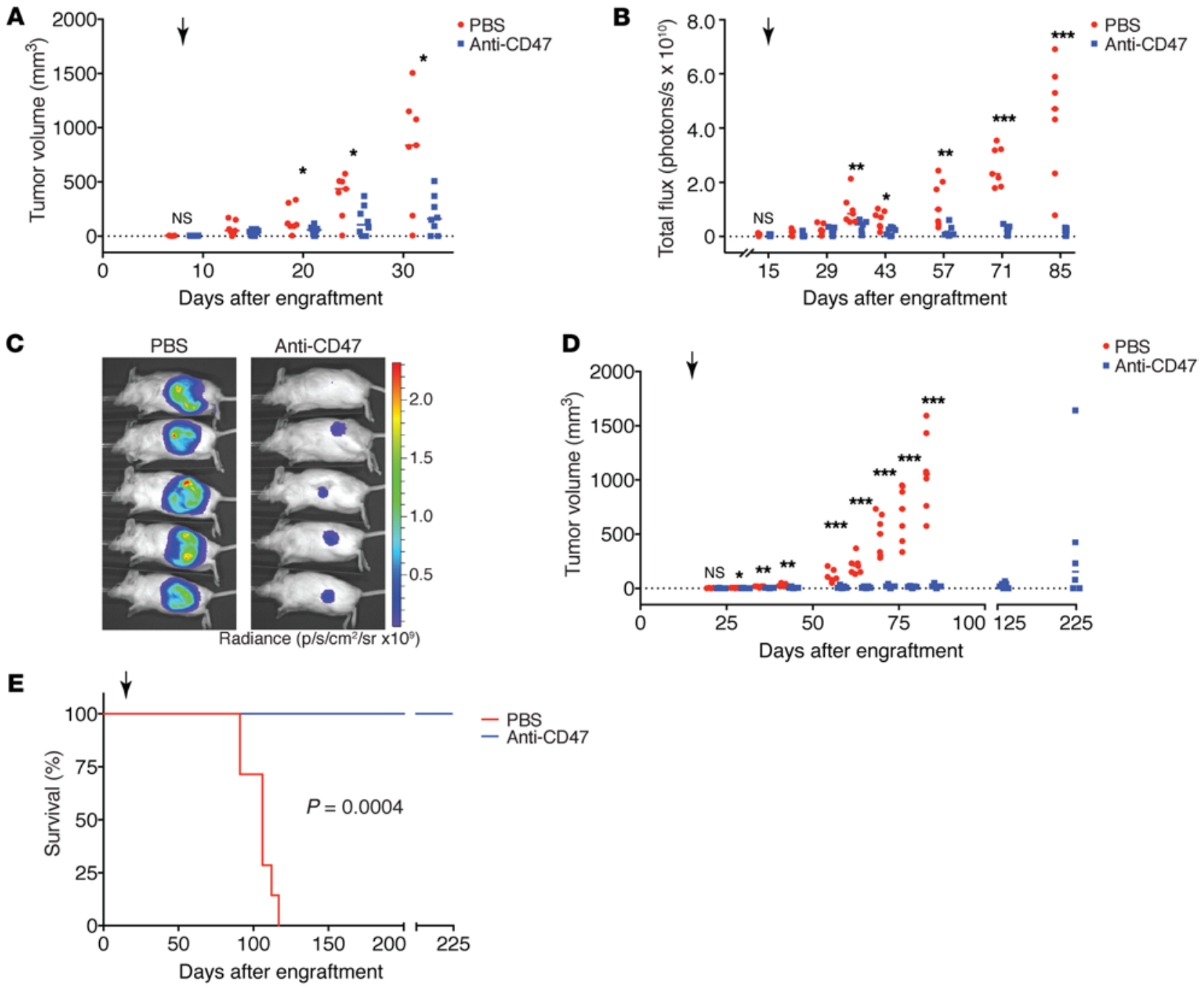

Figure 3. CD47-blocking antibodies inhibit the growth of human SCLC tumors in vivo. (A) Growth of NCI-H82 cells in the subcutaneous tissue of NSC mice. Mice were randomized into groups treated with vehicle control (PBS) or anti-CD47 antibodies (Hu5F9-G4). Growth was evaluated by tumor volume measurements. Points, measurements from independent animals; bars, median. (B) Growth of GFP-luciferase ${ }^{+}$PDX NJH29 tumors in the subcutaneous tissue of NSC mice as evaluated by bioluminescence imaging. Mice were randomized into groups treated with vehicle control (PBS) or anti-CD47 antibodies (Hu5F9-C4). (C) Representative bioluminescence images of $\mathrm{NJH} 29$ tumors on day 85 after engraftment. (D) Growth of $\mathrm{NJH} 29$ tumors as evaluated by tumor volume measurements. (E) Survival of mice bearing PDX NJH29 tumors treated with the indicated therapies. $P=0.0004$ by Mantel-Cox test. (A-E) Black arrows indicate the start of treatment; points indicate measurements from independent animals. Volume measurements at each time point are staggered for clarity. Bars indicate median values. $n=7-8$ mice per treatment cohort. ${ }^{*} P<0.05 ;{ }^{* *} P<0.01 ;{ }^{* *} P<0.001$; ${ }^{* * *} P<0.0001$, Mann-Whitney $U$ test unless otherwise indicated.

cells were resistant to treatment with Hu5F9-G4, indicating no off-target effects of the therapeutic antibody in this model (Supplemental Figure 4D).

CD47 ablation inhibits SCLC tumor growth in immunocompetent mice. Xenograft models enable the evaluation of human tumor growth in vivo, but they are limited in their ability to assess responses in the setting of a fully intact immune system. Therefore, we treated SCLC tumors growing in the lungs of mice in an autochthonous mouse model of SCLC with an anti-mouse CD47 antibody. We observed a trend toward tumor inhibition in the treated mice compared with controls (Supplemental Figure 6, A and B). Because these mice develop over 60 tumors in their lungs (26), which may complicate the analysis of this model, we sought to analyze how single tumors may respond. To this end, we generated a $C d 47$ knockout version of
KP1, a murine SCLC cell line derived from a genetically engineered mouse (27). Compared with the parental KP1 cells, the $C d 47$ knockout variant exhibited increased phagocytosis by mouse macrophages in vitro (Figure 5, B and C). When engrafted into immunocompetent mice, the KP1 Cd47 knockout cell line exhibited significant inhibition of tumor growth (Figure 5D). The KP1 Cd47 knockout cell line also exhibited significant inhibition of tumor growth when engrafted into NSG mice (Figure $5 \mathrm{E}$ ), indicating an important contribution by the myeloid arm of the immune system in mediating these effects.

CD47-blocking therapies stimulate macrophage cytokine secretion. We further examined macrophage activation in response to CD47-blocking therapies by analyzing cytokine secretion. NSG macrophages were cocultured with NCI-H82 cells in the presence or absence of Hu5F9-G4, and supernatants were collected after 
A

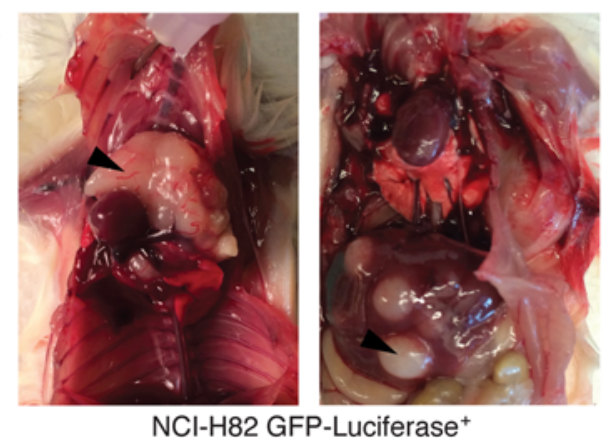

$\mathrm{NCl}$-H82 GFP-Luciferase ${ }^{+}$
B

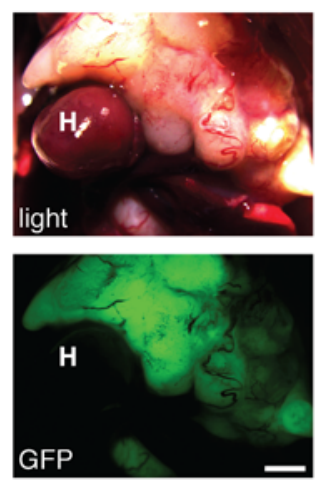

C

PBS
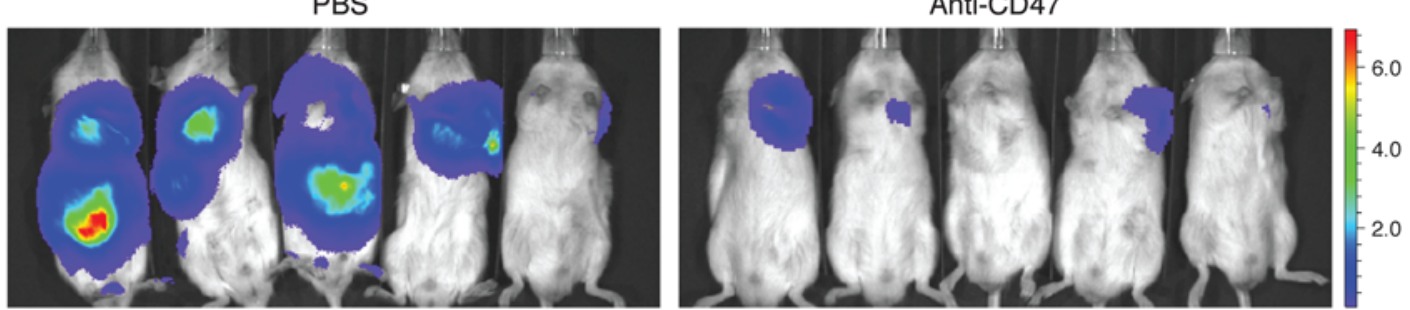

Radiance $\left(\mathrm{p} / \mathrm{s} / \mathrm{cm}^{2} / \mathrm{sr} \times 10^{9}\right)$

D

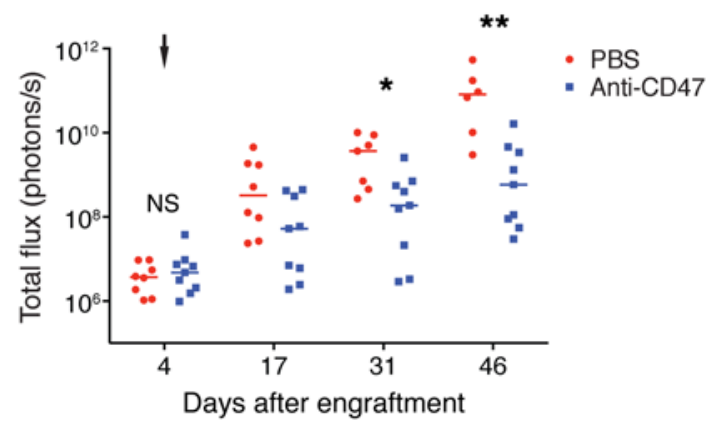

E

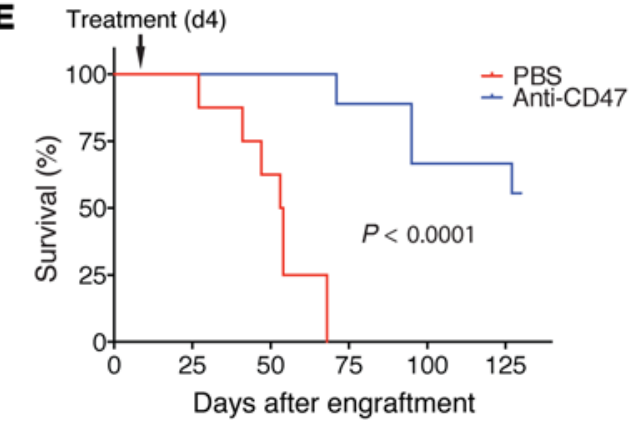

Figure 4. CD47-blocking antibodies are effective in an orthotopic model of SCLC. (A) Representative images of mice injected with SCLC tumor cells showing tumor growth in the thoracic area (left, arrowhead), with some mice developing metastases to the liver (right, arrowhead). (B) Tumor growth monitored postmortem under normal light (top) and under fluorescent light (for GFP expression, bottom). Scale bar: 2 mm. H, heart. (C) Representative bioluminescence images of $\mathrm{NCl}-\mathrm{H} 82$ tumors on day 46 after engraftment in control mice (PBS) and mice treated with CD47-blocking antibodies. (D) Bioluminescence measurements over time. Points represent values from individual mice; bars represent median. Note dropout of mice in PBS cohort over time due to mortality. $n=8-9$ mice per treatment cohort. ${ }^{*} P<0.05 ;{ }^{* *} P<0.01$, Mann-Whitney $U$ test. (E) Survival of mice bearing NCI-H82 tumors treated with the indicated therapies. $P<0.0001$ by Mantel-Cox test. ( $\mathbf{D}$ and $\mathbf{E})$ Arrows indicate start of treatment.

4 hours, then subjected to a multiplexed analysis of 38 mouse cytokines. We identified 5 mouse cytokines that were significantly increased in response to anti-CD47 antibodies: monocyte chemotactic protein 1 (MCP-1, CCL-2), macrophage inflammatory protein 2 (MIP2), GRO- $\alpha$, MIP1b, and IL-6 (Supplemental Figure 7). Interestingly, the macrophages secreted neither IL-10 nor IL12p70 in response to $\mathrm{CD} 47$ blockade in culture, suggesting they may exhibit a phenotype distinct from M1 or M2, as described in the literature (28-30). To identify cytokines secreted in vivo, we engrafted mice with NCI-H82 or NHJ29 cells. We allowed tumors to grow to approximately $1.5 \mathrm{~cm}$ in diameter and then treated the mice with a single dose of vehicle control or anti-CD47 antibody Hu5F9-G4. We collected blood samples just before treatment and 24 hours after treatment and subjected these samples to multiplex analysis of 38 mouse cytokines. From this analysis, we found that MCP-3 was the only cytokine that was significantly increased following treatment with anti-CD47 antibody Hu5F9-G4 in both tumor models (Supplemental Figure 8). No significant increase in MCP-3 was observed in mice without tumors that were treated with anti-CD47 antibody Hu5F9-G4. Together, these assays suggest that the combination of local and systemic cytokine secretion may promote macrophage recruitment and activation to contribute to the efficacy of CD47-blocking therapies.

Comprehensive antibody arrays identify therapeutic targets on $S C L C$. Monoclonal antibodies have proven to be some of the most effective treatments for cancer (31). However, there are few known antibody targets on the surface of SCLC cells (32-35), and there are no clinically approved therapeutic antibodies for SCLC. For this reason, we sought to characterize the surface antigen profile of SCLC cells using comprehensive antibody arrays. To this end, we subjected 4 established SCLC cell lines and the primary SCLC sample PDX NJH29 to analysis using the BioLegend LEGENDScreen array, a comprehensive collection of 332 antibodies to human cell-surface antigens. We identified 39 antigens that were highly expressed on 
A

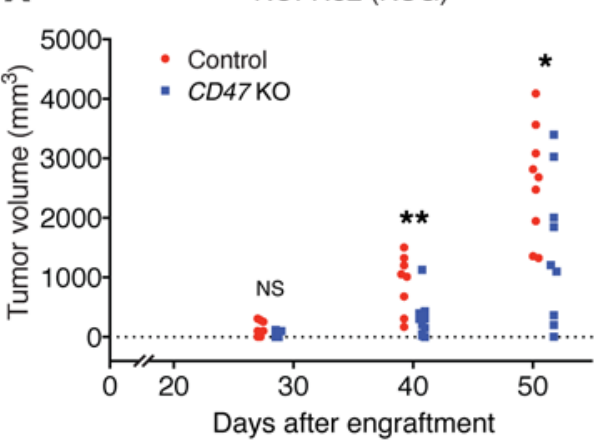

D

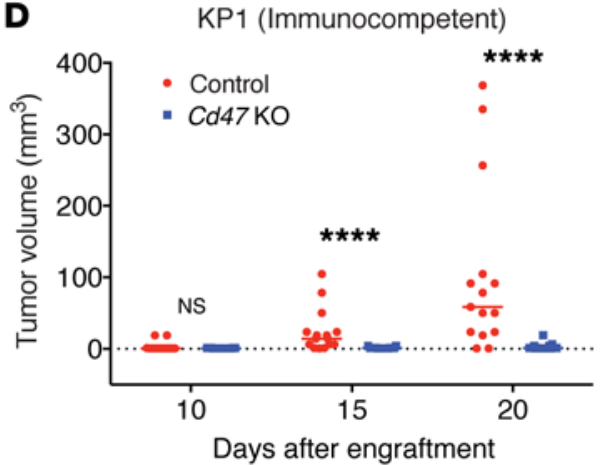

B
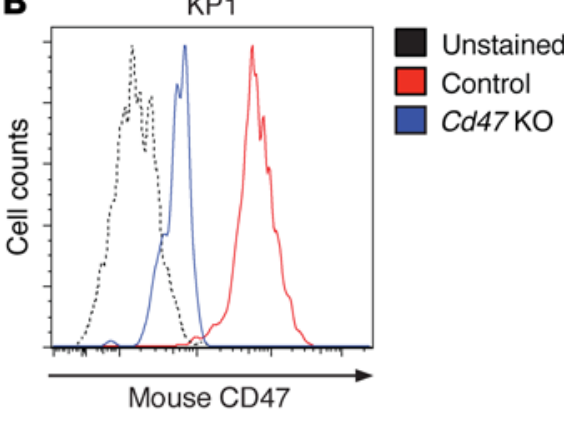

C

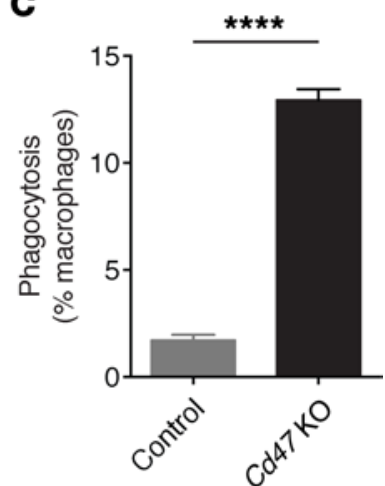

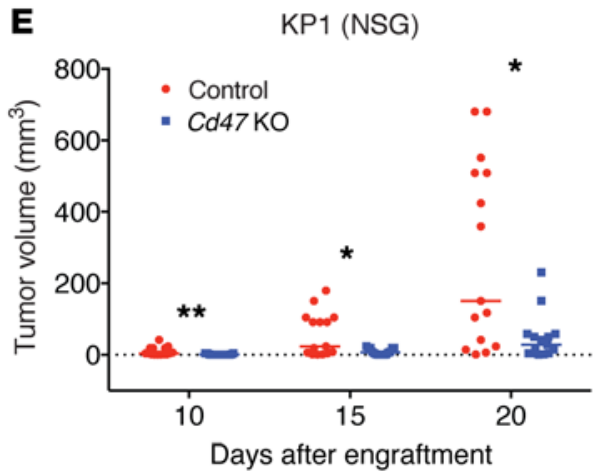

Figure 5. Genetic ablation of $\mathbf{C d 4 7}$ inhibits SCLC growth in vivo. (A) Growth of Cas9 control or CD47 knockout NCI-H82 cells after subcutaneous engraftment in NSC mice. (B-E) Generation of a mouse Cd47 knockout SCLC cell line. KP1 cells were subjected to CRISPR/Cas9 genome editing to create a KP1 Cd47 knockout variant. (B) FACS analysis showing CD47 expression on the surface of KP1 cells (red) compared with KP1 Cd47 knockout cells after 2 rounds of sorting (blue). Dotted black line represents unstained KP1 cells. (C) In vitro phagocytosis assay performed with RFP+ mouse macrophages and KP1 or KP1 Cd47 knockout cells. Data represent mean \pm SD from 3 replicates. ${ }^{* * *} P<0.0001,2$-tailed $t$ test. (D and E) Growth of control or Cd47 knockout mouse KP1 cells in immunocompetent (D) or NSG (E) mice. (A, D, and E) Points indicate measurements from independent animals. Volume measurements at each time point are staggered for clarity. Bars indicate median values. Cohorts consisted of $n=9-10(\mathbf{A})$ or $n=15(\mathbf{D}$ and $\mathbf{E})$ mice. ${ }^{*} P<0.05 ;{ }^{* *} P<0.01 ;{ }^{* * *} P<0.0001$, Mann-Whitney $U$ test $(\mathbf{A}, \mathbf{D}$, and $\mathbf{E})$.

the surface of the SCLC samples (Figure 6A). When we ranked these antigens by their median staining intensity, we found that, strikingly, CD47 was the most intensely staining surface antigen (Figure 6B and Supplemental Table 2). Of note, other immune checkpoint ligands, such as CD80 (B7-1), CD86 (B7-2), PD-L1 (CD274), or PD-L2 (CD273), were not appreciably expressed on the surface of the SCLC samples (Supplemental Table 2); accordingly, very low expression of these cell-surface antigens was found in RNA analyses of cell lines and bulk tumors (Supplemental Figure 1, C and D) (36). Another highly expressed antigen across all samples was CD56 (neural cell adhesion molecule [NCAM]), a known marker of neuroendocrine tumors (34) and a therapeutic target currently under evaluation for $\operatorname{SCLC}(37,38)$, validating our approach. A number of other highly expressed surface antigens were also identified that could potentially be targeted by monoclonal antibody therapies, including CD24 (33), CD29, and CD99 (see below).

As expected, the transcripts coding for the cell-surface molecules detected by flow cytometry were all detectable in SCLC cell lines and bulk tumors, and overall, the correlation between protein expression data measured by flow cytometry and RNA levels measured by microarrays was significant $(r=0.74)$. However, this correlation was not perfect, and outliers included CD47 and NCAM, for which protein levels were substantially greater than expected based on RNA levels (Supplemental Figure 9 and Supplemental
Table 3). These observations underscore the importance of surface antigen profiling methods in identifying therapeutic targets and indicate that the identification of biomarkers such as CD47 and NCAM should not rely exclusively on RNA analysis.

Combining antibodies with CD47 blockade enhances phagocytosis of SCLC. CD47-blocking therapies have been shown to enhance the response of macrophages to monoclonal antibodies (11), but no monoclonal antibodies are clinically approved for the treatment of SCLC. We reasoned that the antigens identified by the LEGENDScreen arrays could serve as therapeutic targets, and we obtained monoclonal antibodies to a number of highly expressed antigens and evaluated their ability to induce phagocytosis in vitro. Specifically, we tested antibodies to CD56 (clones HCD56 and MEM-188), CD24, CD29, and CD99. Additionally, we obtained the sequence for lorvotuzumab, an anti-CD56 antibody being evaluated in clinical trials as an antibody-drug conjugate (38), and we produced it recombinantly as a naked antibody. We tested these antibodies alone and in combination with the high-affinity CD47 antagonist CV1 (10), which blocks CD47 but does not contribute an additional Fc stimulus (Figure 6, C and D). We quantified the ability of these antibodies to induce phagocytosis by human macrophages of 2 different SCLC cell lines, NCI-H82 (Figure 6C) and NCI-H524 (Figure 6D). Of the 3 anti-CD56 antibod- 
A

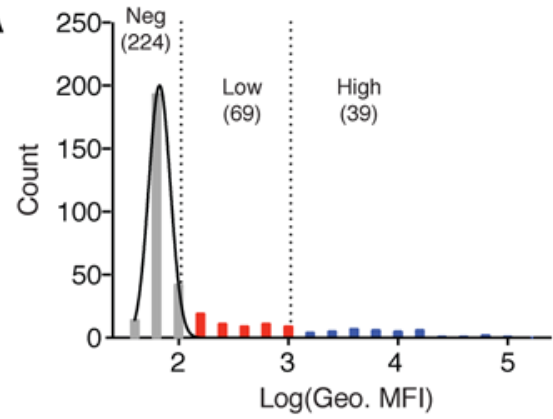

C

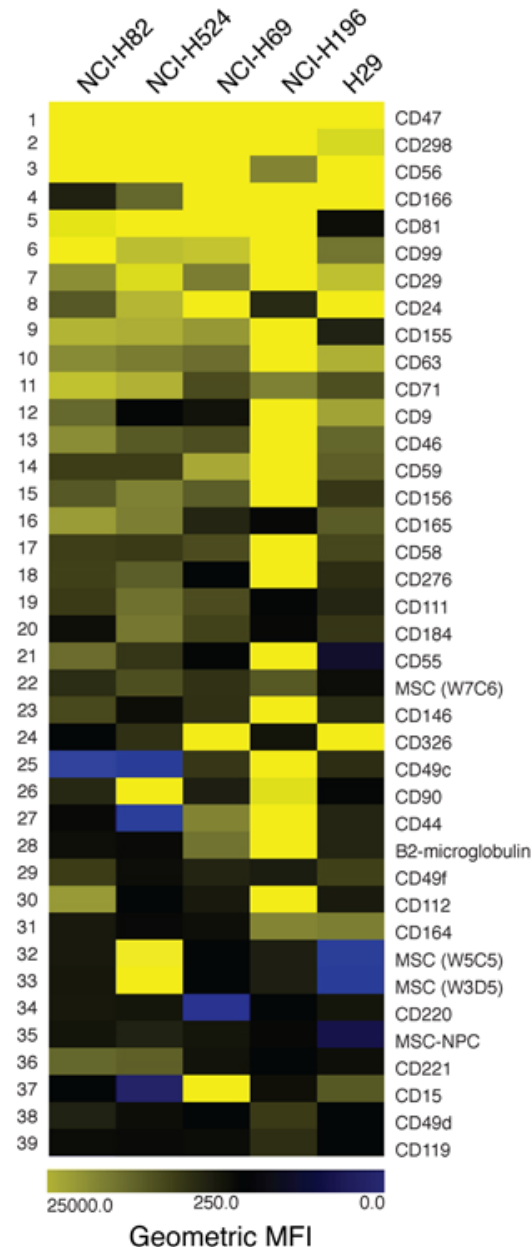

B

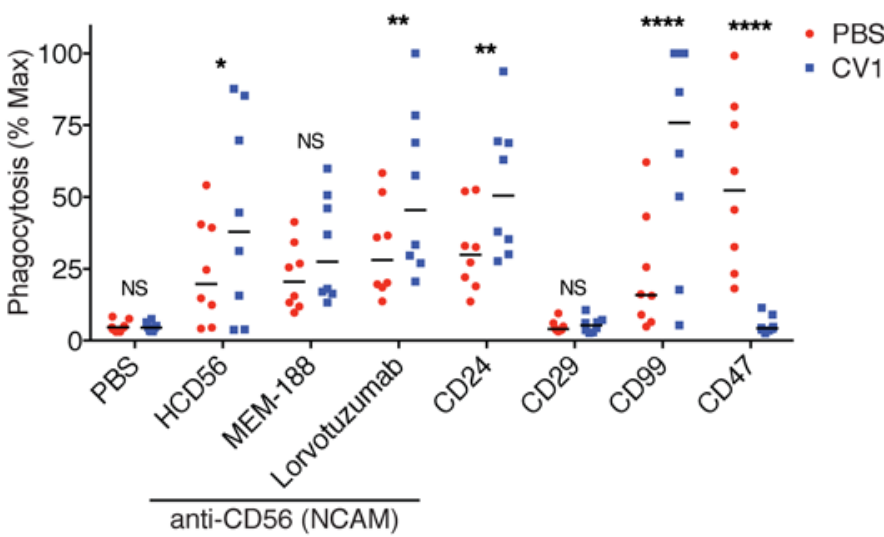

D

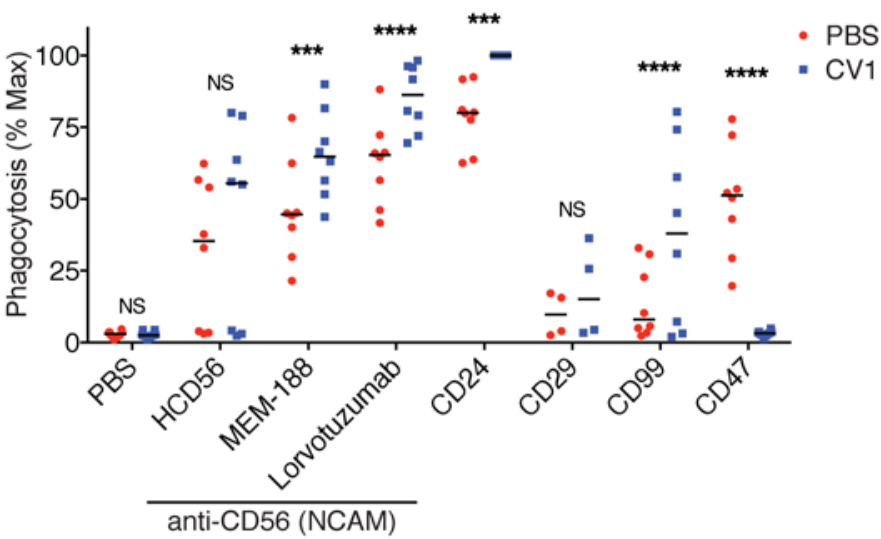

E

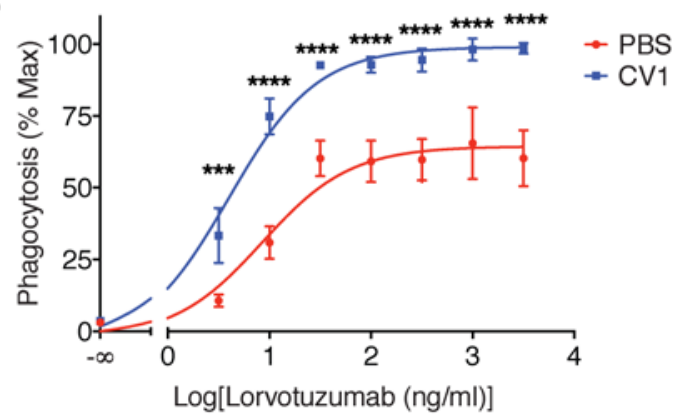

Figure 6. Comprehensive antibody screening identifies targets on SCLC for combination therapy with high-affinity SIRP $\alpha$ variants. Antigen expression on the surface of 4 SCLC cell lines and patient sample NJH29 was assessed by flow cytometry using a collection of 332 antibodies targeting surface antigens. (A) Histogram depicting geometric mean fluorescence intensity (MFI) of all antibodies screened for binding. Median values for each antibody across all 5 samples were fit to Gaussian distribution (black line). Negative antigens (gray), low antigens (red), and high antigens (blue) defined based on MFI thresholds as described in Supplemental Methods (see Supplemental Table 2 for full antigen list). The number of antigens in each category is indicated in parentheses. Geo, geometric. (B) Ranked list of the 39 antigens identified as high based on median MFI across all 5 SCLC samples. (C-D) Phagocytosis of NCl-H82 cells (C) and $\mathrm{NCl}-\mathrm{H} 524$ cells (D) in response to tumor-binding antibodies alone (red) or in combination with high-affinity SIRP $\alpha$ variant CV1 monomer (blue). Points, measurements from individual donors; bars, median values. Three clones of anti-CD56 (NCAM) antibodies were tested, as well as antibodies to CD24, CD29, CD99, and CD47 (Hu5F9-C4). (E) Phagocytosis of NCI-H82 SCLC cells in response to varying concentrations of the anti-CD56 antibody lorvotuzumab alone (red) or in combination with the high-affinity SIRP $\alpha$ variant CV1 monomer (blue). Data represent mean \pm SD. (C-E) Phagocytosis was normalized to the maximal response by each donor $\left(n=4-8\right.$ donors). ${ }^{*} P<0.05$; ${ }^{* *} P<0.01$; ${ }^{* *} P<0.001 ;{ }^{* * *} P<0.0001,2$-way ANOVA with Šidák correction.

ies tested, we found that lorvotuzumab was able to produce the greatest increase in phagocytosis, and this effect was significantly enhanced by combination with CV1. Antibodies to CD24 or CD99 were also able to induce phagocytosis that was comparable to or exceeded that of treatment with anti-CD47 antibody Hu5F9-G4. As expected, no increase in phagocytosis was observed when anti-CD47 antibody Hu5F9-G4 was combined with CV1, since CV1 competes for the same binding 
interface and binds with extremely high affinity. Interestingly, the anti-CD29 antibody was not able to induce phagocytosis even in combination with CV1, an important demonstration that additional factors, such as surface-binding geometry or the ability to engage Fc receptors, may modify the response of macrophages to therapeutic antibodies.

Since lorvotuzumab has been under evaluation as a therapeutic agent for SCLC (38), we investigated its ability to induce phagocytosis over a varying range of concentrations. Treatment with lorvotuzumab alone produced a dose-response relationship for inducing macrophage phagocytosis. Importantly, we found that for each lorvotuzumab concentration tested, the addition of CV1 produced a greater degree of phagocytosis (Figure 6E). Treatment with CV1 could increase both the maximal efficacy and the potency of lorvotuzumab in SCLC, as previously observed when CV1 was combined with rituximab, trastuzumab, and cetuximab in other contexts (10). Thus, combining therapeutic antibodies with CD47-blocking strategies may represent an alternative method for enhancing the efficacy of antibodies.

\section{Discussion}

In conclusion, we observed widespread CD47 expression in SCLC, including at different stages of cancer development and following chemotherapy, and we show that CD47 is a genuine immunotherapeutic target for SCLC. Macrophages contribute to this therapeutic response, but CD47 blockade can also activate other myeloid immune subsets, including neutrophils and dendritic cells, and may initiate adaptive immune responses (12, 39, 40). Since Hu5F9-G4 is under investigation in clinical trials for human solid malignancies and acute myelogenous leukemia, our findings provide scientific justification for further evaluation of CD47blocking therapies in patients with SCLC.

In our histological analysis of 79 SCLC patient samples, we observed a positive association between macrophage infiltration and tumor stage. A prior study observed greater macrophage infiltration in early stage tumors (15). Regardless of this difference, both studies conclusively show that a substantial number of macrophages are present in many SCLC tumors. The degree of macrophage infiltration and its relationship to CD47 expression on tumors may predict which patients respond best to CD47 therapies, and this subject warrants further investigation in preclinical and clinical studies.

Macrophages in SCLC tumors expressed CD163, a marker of immunosuppressive "M2" macrophages that may promote tumor growth. However, M2 macrophages may exhibit a greater capacity for phagocytosis in response to therapeutic antibody relative to proinflammatory M1 macrophages (41). A recent report has also demonstrated that both M1 and M2 macrophages perform phagocytosis in response to anti-CD47 treatment (42). Furthermore, treatment with anti-CD47 antibodies converted tumor-associated macrophages to an M1-like state in vivo (42). The dichotomy of M1 versus M2 polarization underrepresents the complexity of macrophage activation, which is dynamic and fluid with a plethora of potential activation states $(30,43)$. Indeed, based on cytokine secretion in response to anti-CD47 therapy, the macrophages in our in vitro studies did not clearly fit common descriptions of either M1 or M2 activation. Further investigations are needed to determine whether certain polarizing stimuli can enhance macrophage responses to CD47-blocking therapies.

Interestingly, our SCLC models and prior studies have shown increased macrophage infiltration into tumors following treatment with CD47-blocking therapies (10). Macrophage recruitment or proliferation may be an additional mechanism that contributes to the efficacy of these therapies. Our studies on cytokine secretion in vitro and in vivo suggest a number of chemokines that may act by promoting monocyte or macrophage infiltration. Although this finding requires more investigation to definitively identify factors and demonstrate a causal relationship, this is an attractive hypothesis to explain the robust antitumor efficacy that is observed in many treatment models. If true, this finding could also suggest therapeutic combinations with factors that further promote macrophage recruitment or activation in tumors.

In many models, CD47 blockade does not completely eliminate tumors $(9,10,14)$. As tumors increase in size, the penetrance of antibody therapies decreases given their high molecular weight (44). Thus, persistent growth may reflect pharmacokinetic limitations in the setting of a rapidly proliferating tumor rather than selection for resistance at the genetic or epigenetic level. Nevertheless, mechanisms of resistance to CD47-blocking therapies could depend on a variety of factors, including cell size, the tumor microenvironment, and target expression. In our studies, loss of CD47 made SCLC cells resistant to anti-CD47 antibodies, but we did not observe this resistance mechanism developing in situ following treatment. Other studies have found that cancer specimens intrinsically differ in their susceptibility to phagocytosis $(8-10,14)$. In most cases, CD47 blockade or knockout is not sufficient to induce phagocytosis, but requires an additional prophagocytic stimulus, such as opsonizing antibodies (10). Mouse KP1 cells exhibited a moderate increase in phagocytosis with $C d 47$ ablation alone, suggesting these cells may be more prone to phagocytosis. In contrast, we identified one human SCLC cell line (NCI-H196) that exhibited no significant increase in phagocytosis even when treated with anti-CD47 antibodies. These responses may be influenced by differences in intrinsic prophagocytic signals or inhibitory signals on the surface of the cancer cells. For example, CD47 expression levels between SCLC tumors and relative to other tumor types need to be better quantified, as these levels may dictate some of the response to CD47-blocking strategies. In addition, calreticulin has been identified as one such prophagocytic signal and may play particular importance in the malignant conversion of myelodysplastic syndrome to acute myelogenous leukemia $(45,46)$. The identification of redundant inhibitory signals remains an active area of investigation. These signals may have implications for resistance to CD47-blocking therapies and may also serve as biomarkers that identify patients who will respond best to treatment.

Emerging studies indicate that both the innate and adaptive immune systems may contribute to the efficacy of CD47-blocking therapies. The CD47/SIRP $\alpha$ axis may be an early checkpoint in immune activation, regulating phagocytosis and antigen uptake to then promote antigen presentation to $\mathrm{T}$ cells. Both macrophages and dendritic cells may mediate this link in response to CD47 blockade $(39,40)$. Furthermore, a recent study demonstrated that CD47-blocking therapies may enhance the efficacy of other immune checkpoint inhibitors, such as agents targeting 
the PD-1/PD-L1 axis (47). We found that $C d 47$ ablation was effective at inhibiting tumor growth in both immunocompromised and immunocompetent models, where immunosuppressive cells such as Treg could limit antitumor responses (48). SCLC exhibits a high mutation burden (49), likely due to its association with heavy tobacco use and frequent inactivation of p53 and pRB (50); therefore, it may be particularly responsive to therapies that engage the adaptive immune system. Therapies targeting $\mathrm{T}$ cell immune checkpoints are now being tested in $\operatorname{SCLC}(51,52)$ and may cooperate with CD47-blocking approaches $(40,47)$.

Finally, our approach to identifying SCLC surface antigens can be applied to other types of cancer and, in the future, could be used to assemble oligoclonal cocktails of antibodies that could be used to simulate the natural humoral immune response against foreign pathogens or cells. These cocktails could be combined with CD47-blocking therapies and other immunotherapies or targeted therapies (53) to mount an effective immune response against cancer cells. This strategy could serve as the foundation to personalized immunotherapeutic regimens that could be tailored for individual patients or different cancer types.

\section{Methods}

Detailed Methods can be found in Supplemental Methods.

Study approval. Mice were maintained in a barrier facility under the care of the Stanford Veterinary Services Center. All animal studies were approved by the Administrative Panel on Laboratory Animal Care at Stanford University.

Statistics. Macrophage infiltration scores and tumor stage were analyzed by Spearman correlation. In vitro phagocytosis was analyzed by 2-tailed $t$ test or 2-way ANOVA with Šidák correction. Tumor volumes and bioluminescent imaging were assessed by Mann-Whitney $U$ test or 1-way ANOVA with Holm-Šidák correction for multiple comparisons. Survival was analyzed by Mantel-Cox test. Assessment of $\mathrm{F} 4 / 80^{+}$macrophage infiltration following treatment was performed using an unpaired $t$ test. Lung weights in genetically engineered mouse models were assessed by $t$ test. Cytokine secretion was analyzed by 2-way ANOVA with Šidák correction for multiple comparisons. Statistical analysis was performed using Prism (GraphPad). P values of 0.05 or less were considered statistically significant.

Large data sets. RNA-seq data from 41 human primary SCLC tumors were previously published $(18,19)$ and were deposited in the NCBI's Gene Expression Omnibus (GEO GSE69091). The October 17, 2012, release of the CCLE Cell Line Gene Expression data (Cancer Cell Line Encyclopedia; ref. 54) was downloaded from the Broad Institute website. Atomic coordinates of the Hu5F9-G4/CD47-ECD crystal structure have been deposited in the Protein Data Bank (PDB 5IWL).

\section{Author contributions}

KW, ILW, and JS designed research. KW and JS wrote the manuscript. KW and JS prepared the figures with the other authors. KW, NSJ, JG, SML, and JS performed CD47 protein or RNA expression analysis. KW and PJS performed in vitro phagocytosis assays. DW, KJ, and KCG performed crystallographic analysis. KW, AMR, JL, and RM produced or contributed therapeutic reagents. KW, NSJ, PJS, SC, AKV, JPV, JSL, DY, GS, TN, HG, and MMW performed in vivo experiments. KW, NSJ, and RLM performed LegendSCREEN profiling analysis. $\mathrm{KW}, \mathrm{HV}$, and MVDR performed histological analysis. KW, JSL, DY, AB, AMR, FT, JTP, EEG, LAM, EDS, CD, RKT, and CMR generated or contributed reagents and specimens. All authors critically reviewed the manuscript.

\section{Acknowledgments}

The authors wish to thank members of the Weissman and Sage labs for helpful advice and discussions. The authors thank the CD47 Disease Team for discussions and providing anti-CD47 antibodies. The authors thank R. Levy, B. Mitchell, H. Kohrt, J. Shizuru, T. Storm, L. Jerabek, N. Fernhoff, H. Contreras-Trujillo, A. McCarty, K. McKenna, S. Willingham, P. Ho, S. Prohaska, and M. Howard for technical assistance, discussions, and reagents. The authors thank Y. Rosenberg-Hasson, I. Herschmann, H. Maecker, and the staff of the Stanford Human Immune Monitoring Center as well as Marissa Mattar and Xiaodong Huang of the MSK Antitumor Assessment Core Facility for their technical expertise. The authors acknowledge that gene expression analyses were performed with RNA-seq data partly generated by Genentech/gRED and published in ref. 17. The content of this manuscript is solely the responsibility of the authors. Research reported in this publication was supported by the National Cancer Institute (F30 CA168059 to K. Weiskopf, P01 CA139490 to I.L. Weissman, and P30 CA008748 to E. de Stanchina), the Geoffrey Beene Cancer Research Center (to E. de Stanchina and J.T. Poirier), the Stanford Medical Scientist Training Program (NIH-GM07365 to K. Weiskopf and A.M. Ring), the Stanford University SPARK Program (to K. Weiskopf and A.M. Ring), a California TRDRP post-doctoral fellowship (to N.S. Jahchan), the Deutsche Forschungsgemeinschaft (VO 1976/1 to A.K. Volkmer and through TH1386/3-1 to R.K. Thomas), the German Cancer Aid (Deutsche Krebshilfe) as part of the SCLC genome sequencing consortium (grant ID: 109679 to R.K. Thomas), the German Ministry of Science and Education (BMBF) as part of the e:Med program (01ZX1303A to R.K. Thomas), the Howard Hughes Medical Institute (K.C. Garcia), the Joseph \& Laurie Lacob Gynecologic/Ovarian Cancer Fund (I.L. Weissman), the Virginia and D.K. Ludwig Fund for Cancer Research (I.L. Weissman), the Lucile Packard Foundation for Children's Health (J. Sage), the LUNGevity Foundation (J. Sage, J.T. Poirier), an anonymous donors fund, the Siebel Stem Cell Institute, and the Thomas and Stacey Siebel Foundation (I.L. Weissman, K.C. Garcia). J. Sage is the Harriet and Mary Zelencik Scientist in Children's Cancer and Blood Diseases. Use of the Stanford Synchrotron Radiation Lightsource, SLAC National Accelerator Laboratory, is supported by the US Department of Energy, Office of Science, Office of Basic Energy Sciences under contract no. DEAC02-76SF00515. The SSRL Structural Molecular Biology Program is supported by the Department of Energy Office of Biological and Environmental Research, and by the NIH, National Institute of General Medical Sciences (including P41GM103393).

Address correspondence to: Kipp Weiskopf, Stanford University, 265 Campus Drive, SIM1 G3155, Stanford, California 94305-5457, USA. Phone: 650.723.6520; E-mail: kippw@stanford.edu. Or to: Irving L. Weissman, Stanford University, 265 Campus Drive, SIM1 G3167, Stanford, California 94305-5457, USA. Phone: 650.723.6520; E-mail: irv@stanford.edu. Or to: Julien Sage, Stanford University, 265 Campus Drive, SIM1 G2078, Stanford, California, 94305-5457, USA. Phone: 650.724.9246; E-mail: julsage@stanford.edu. 
1. Park KS, et al. Characterization of the cell of origin for small cell lung cancer. Cell Cycle. 2011;10(16):2806-2815.

2. Sutherland KD, Proost N, Brouns I, Adriaensen D, Song JY, Berns A. Cell of origin of small cell lung cancer: inactivation of Trp53 and rb1 in distinct cell types of adult mouse lung. Cancer Cell. 2011;19(6):754-764.

3. van Meerbeeck JP, Fennell DA, De Ruysscher DK. Small-cell lung cancer. Lancet. 2011;378(9804):1741-1755.

4. Byers LA, Rudin CM. Small cell lung cancer: where do we go from here? Cancer. 2015;121(5):664-672.

5. Pardoll DM. The blockade of immune checkpoints in cancer immunotherapy. Nat Rev Cancer. 2012;12(4):252-264.

6. Page DB, Postow MA, Callahan MK, Allison JP, Wolchok JD. Immune modulation in cancer with antibodies. Ann Rev Med. 2014;65:185-202.

7. Jaiswal S, et al. CD47 is upregulated on circulating hematopoietic stem cells and leukemia cells to avoid phagocytosis. Cell. 2009;138(2):271-285.

8. Majeti R, et al. CD47 is an adverse prognostic factor and therapeutic antibody target on human acute myeloid leukemia stem cells. Cell. 2009;138(2):286-299.

9. Willingham SB, et al. The CD47-signal regulatory protein alpha (SIRPa) interaction is a therapeutic target for human solid tumors. Proc Natl Acad Sci U S A. 2012;109(17):6662-6667.

10. Weiskopf K, et al. Engineered SIRPalpha variants as immunotherapeutic adjuvants to anticancer antibodies. Science. 2013;341(6141):88-91.

11. Weiskopf K, Weissman IL. Macrophages are critical effectors of antibody therapies for cancer. MAbs. 2015;7(2):303-310.

12. Zhao XW, et al. CD47-signal regulatory proteinalpha (SIRPalpha) interactions form a barrier for antibody-mediated tumor cell destruction. Proc Natl Acad Sci U S A. 2011;108(45):18342-18347.

13. Theocharides AP, et al. Disruption of SIRPalpha signaling in macrophages eliminates human acute myeloid leukemia stem cells in xenografts. JExp Med. 2012;209(10):1883-1899.

14. Chao MP, et al. Anti-CD47 antibody synergizes with rituximab to promote phagocytosis and eradicate non-Hodgkin lymphoma. Cell. 2010;142(5):699-713.

15. Eerola AK, Soini Y, Paakko P. A high number of tumor-infiltrating lymphocytes are associated with a small tumor size, low tumor stage, and a favorable prognosis in operated small cell lung carcinoma. Clin Cancer Res. 2000;6(5):1875-1881.

16. Peifer $\mathrm{M}$, et al. Integrative genome analyses identify key somatic driver mutations of small-cell lung cancer. Nat Genet. 2012;44(10):1104-1110.

17. Rudin CM, et al. Comprehensive genomic analysis identifies SOX2 as a frequently amplified gene in small-cell lung cancer. Nat Genet. 2012;44(10):1111-1116.

18. Pedersen N, et al. Transcriptional gene expression profiling of small cell lung cancer cells. Cancer Res. 2003;63(8):1943-1953.

19. Hodgkinson CL, et al. Tumorigenicity and genetic profiling of circulating tumor cells in small-cell lung cancer. Nat Med. 2014;20(8):897-903.

20. Liu J, et al. Pre-clinical development of a humanized anti-CD47 antibody with anti-cancer therapeutic potential. PLoS One. 2015;10(9):e0137345.

21. Hatherley D, Graham SC, Turner J, Harlos K, Stuart DI, Barclay AN. Paired receptor specificity explained by structures of signal regulatory proteins alone and complexed with CD47. Mol Cell. 2008;31(2):266-277.

22. Shultz LD, et al. Human lymphoid and myeloid cell development in NOD/LtSz-scid IL2R gamma null mice engrafted with mobilized human hemopoietic stem cells. Jimmunol. 2005;174(10):6477-6489.

23. Leong TL, et al. Genomic characterisation of small cell lung cancer patient-derived xenografts generated from endobronchial ultrasound-guided transbronchial needle aspiration specimens. PLoS One. 2014;9(9):e106862.

24. Daniel VC, et al. A primary xenograft model of small-cell lung cancer reveals irreversible changes in gene expression imposed by culture in vitro. Cancer Res. 2009;69(8):3364-3373.

25. Nakazawa K, et al. Specific organ metastases and survival in small cell lung cancer. Oncol Lett. 2012;4(4):617-620.

26. Schaffer BE, et al. Loss of $\mathrm{p} 130$ accelerates tumor development in a mouse model for human small-cell lung carcinoma. Cancer Res. 2010;70(10):3877-3883.

27. Park KS, et al. A crucial requirement for Hedgehog signaling in small cell lung cancer. Nat Med. 2011;17(11):1504-1508.

28. Mantovani A, Biswas SK, Galdiero MR, Sica A, Locati M. Macrophage plasticity and polarization in tissue repair and remodelling.J Pathol. 2013;229(2):176-185.

29. Sica A, Mantovani A. Macrophage plasticity and polarization: in vivo veritas. JClin Invest. 2012;122(3):787-795.

30. Murray PJ, et al. Macrophage activation and polarization: nomenclature and experimental guidelines. Immunity. 2014;41(1):14-20.

31. Sliwkowski MX, Mellman I. Antibody therapeutics in cancer. Science. 2013;341(6151):1192-1198.

32. Fargion S, et al. Heterogeneity of cell surface antigen expression of human small cell lung cancer detected by monoclonal antibodies. Cancer Res. 1986;46(5):2633-2638.

33. Jackson D, Waibel R, Weber E, Bell J, Stahel RA. CD24, a signal-transducing molecule expressed on human B cells, is a major surface antigen on small cell lung carcinomas. Cancer Res. 1992;52(19):5264-5270.

34. Aletsee-Ufrecht MC, Langley K, Rotsch M, Havemann K, Gratzl M. NCAM: a surface marker for human small cell lung cancer cells. FEBS Lett. 1990;267(2):295-300.

35. Teicher BA. Targets in small cell lung cancer. Biochem Pharmacol. 2014;87(2):211-219.

36. Schultheis AM, et al. PD-L1 expression in smal cell neuroendocrine carcinomas. Eur J Cancer. 2015;51(3):421-426.

37. Lambert JM. Drug-conjugated monoclonal antibodies for the treatment of cancer. Curr Opin Pharmacol. 2005;5(5):543-549.
38. Whiteman KR, et al. Lorvotuzumab mertansine, a CD56-targeting antibody-drug conjugate with potent antitumor activity against small cell lung cancer in human xenograft models. MAbs. 2014;6(2):556-566.

39. Tseng D, et al. Anti-CD47 antibody-mediated phagocytosis of cancer by macrophages primes an effective antitumor T-cell response. Proc Natl Acad Sci U S A. 2013;110(27):11103-11108.

40. Liu X, et al. CD47 blockade triggers T cell-mediated destruction of immunogenic tumors. Nat Med. 2015;21(10):1209-1215.

41. Leidi M, et al. M2 macrophages phagocytose rituximab-opsonized leukemic targets more efficiently than $\mathrm{m} 1$ cells in vitro. J Immunol. 2009;182(7):4415-4422.

42. Zhang M, et al. Anti-CD47 Treatment stimulates phagocytosis of glioblastoma by M1 and M2 polarized macrophages and promotes M1 polarized macrophages in vivo. PLOS ONE. 2016;11(4):e0153550.

43. Martinez FO, Gordon S. The M1 and M2 paradigm of macrophage activation: time for reassessment. F1000Prime Rep. 2014;6:13.

44. Beckman RA, Weiner LM, Davis HM. Antibody constructs in cancer therapy: protein engineering strategies to improve exposure in solid tumors. Cancer. 2007;109(2):170-179.

45. Chao MP, et al. Calreticulin is the dominant prophagocytic signal on multiple human cancers and is counterbalanced by CD47. Sci Transl Med. 2010;2(63):63ra94.

46. Pang WW, et al. Hematopoietic stem cell and progenitor cell mechanisms in myelodysplastic syndromes. Proc Natl Acad Sci U S A. 2013;110(8):3011-3016.

47. Sockolosky JT, et al. Durable antitumor responses to $\mathrm{CD} 47$ blockade require adaptive immune stimulation [published online ahead of print April 18, 2016]. Proc Natl Acad Sci U S A. doi:10.1073/ pnas.1604268113.

48. Marabelle A, et al. Depleting tumor-specific Tregs at a single site eradicates disseminated tumors. J Clin Invest. 2013;123(6):2447-2463.

49. Alexandrov LB, et al. Signatures of mutational processes in human cancer. Nature. 2013;500(7463):415-421.

50. George J, et al. Comprehensive genomic profiles of small cell lung cancer. Nature. 2015;524(7563):47-53.

51. Reck M, et al. Ipilimumab in combination with paclitaxel and carboplatin as first-line therapy in extensive-disease-small-cell lung cancer: results from a randomized, double-blind, multicenter phase 2 trial. Ann Oncol. 2013;24(1):75-83

52. Brahmer JR, Pardoll DM. Immune checkpoint inhibitors: making immunotherapy a reality for the treatment of lung cancer. Cancer Immunol Res. 2013;1(2):85-91.

53. Wargo JA, Cooper ZA, Flaherty KT. Universes collide: combining immunotherapy with targeted therapy for cancer. Cancer Discov. 2014;4(12):1377-1386.

54. Barretina J, et al. The Cancer Cell Line Encyclopedia enables predictive modelling of anticancer drug sensitivity. Nature. 2012;483(7391):603-607. 